\title{
CURVATURE ESTIMATES FOR SUBMANIFOLDS IMMERSED INTO HOROBALLS AND HOROCYLINDERS
}

\author{
G. PACELLI BESSA, JORGE H. DE LIRA, STEFANO PIGOLA, \\ AND ALBERTO G. SETTI
}

\begin{abstract}
We prove mean and sectional curvature estimates for submanifolds confined into either a horocylinder of $N \times L$ or a horoball of $N$, where $N$ is a Cartan-Hadamard manifold with pinched curvature. Thus, these submanifolds behave in many respects like submanifolds immersed into compact balls and into cylinders over compact balls. The proofs rely on the Hessian comparison theorem for the Busemann function.
\end{abstract}

\section{INTRODUCTION}

A classical problem in Riemanninan geometry is to obtain curvature estimates for submanifolds under extrinsic constraints. Jorge and Xavier [7], showed that any complete $m$-dimensional Riemannian manifold $M$ with scalar curvature bounded below isometrically immersed into a normal ball $B_{N}(R)$ of a Riemannian manifold $N$ of radius $R$ has mean curvature of $M$ satisfying

$$
\sup _{M}|\mathbf{H}| \geq\left\{\begin{array}{cl}
\sqrt{A} \operatorname{coth}(\sqrt{A} R) & \text { if } \quad K_{N} \leq-A \text { on } B_{N}(R), A>0 \\
\frac{1}{R} & \text { if } \quad K_{N} \leq 0 \text { on } B_{N}(R) \\
\sqrt{A} \cot (\sqrt{A} R) & \text { if } \quad K_{N} \leq A \text { on } B_{N}(R) \text { and } A R<\pi / 2, A>0 .
\end{array}\right.
$$

2010 Mathematics Subject Classification. 53C42, 58J50.

Key words and phrases. Curvature estimates, submanifolds, Cartan-Hadamard manifolds, horoballs, horocylinders.

The authors are grateful to the anonymous referee for a very careful reading of the paper. The third and the fourth authors acknowledge partial support by the Italian Miur, Prin 2010-2011 Varietà reali e complesse: Geometria, topologia e Analisi Armonica, unit locale di Milano-Bicocca, and by INdAM-GNAMPA. They also thank the Departamento de Matemática da Universidade Federal do Ceará, where this work was carried out, for its warm hospitality. The forth author is grateful to CAPES-Ciência Sem Fronteiras, grant 400794/2012-8, for financial support. 
This result was then generalized relaxing the scalar curvature condition [8], and ultimately shown to hold provided $M$ is stochastically complete, [11], [12]. As a result, there are no minimal bounded immersions of a stochastically complete manifold into a Cartan-Hadamard manifold.

In another direction the result was extended even to the case of immersions into cylinders $B_{N}(R) \times \mathbb{R}^{\ell}, \ell<m$, in a product manifold [1].

In our first theorem we prove mean curvature estimates valid for immersions of a stochastically complete manifold into (suitable) cylinders over unbounded bases. More precisely, let $N$ be an $n$-dimensional CartanHadamard manifold and let $\sigma$ be a ray in $N$, with associated Busemann function $b_{\sigma}$. The horoball in $N$ determined by $\sigma$ is the set

$$
\mathscr{B}_{\sigma, R}^{n}=\left\{b_{\sigma} \leq R\right\}, \quad R>0,
$$

and, if $L$ is an arbitrary $\ell$-dimensional manifold, we say that the region

$$
\mathscr{B}_{\sigma, R}^{n, \ell}=\left\{b_{\sigma} \leq R\right\} \times L
$$

is a (generalized solid) horocylinder in $N \times L$. With this notation we have

Theorem 1.1. Let $\left(N, g_{N}\right)$ be an $n$-dimensional Cartan-Hadamard manifold with sectional curvature satisfying $-B \leq \operatorname{Sec}_{N} \leq-A$ and let $\sigma:[0,+\infty) \rightarrow$ $N$ be a ray of $N$. Let $\left(L, g_{L}\right)$ be any $\ell$-dimensional Riemannian manifold and let $f=\left(f_{N}, f_{L}\right): \Sigma \hookrightarrow N \times L$ be an m-dimensional isometric immersion with mean curvature vector field $\mathbf{H}$. Assume that $m>\ell$. If $\Sigma$ is stochastically complete and $f(\Sigma)$ is contained in the horocylinder $\mathscr{B}_{\sigma, R}^{n, \ell}$, then

$$
\sup _{\Sigma}|\mathbf{H}| \geq \frac{m-\ell}{m} \sqrt{A}
$$

We explicitly note that the result remains true when the fibre $L$ degenerates to a 0-dimensional point and, hence, the horocylinder reduces to a horoball.

Since bounded mean curvature submanifolds properly immersed into a complete ambient manifold of bounded sectional curvature are stochastically complete, see for instance [12], as a corollary we have

Theorem 1.2. Let $\left(N, g_{N}\right)$ be an n-dimensional Cartan-Hadamard manifold with sectional curvature satisfying $-B \leq \operatorname{Sec}_{N} \leq-A<0$ and let $\left(L, g_{L}\right)$ be any complete $\ell$-dimensional Riemannian manifold with sectional curvature $\operatorname{Sec}_{L} \geq-C^{2}$, where A, B, C are positive constants. If $f: \Sigma \hookrightarrow N \times L$ is an $m$ dimensional properly immersed submanifold with $f(\Sigma)$ inside a horocylinder of $N \times L$ and $m>\ell$ then the mean curvature vector $\mathbf{H}$ of the immersion satisfies

$$
\sup _{\Sigma}|\mathbf{H}| \geq \frac{m-\ell}{m} \sqrt{A} .
$$


Our second result is the following sectional curvature lower estimate in the spirit of the classical theorem by Jorge-Koutroufiotis, [6]. We point out that, although it is stated for submanifolds in a horoball, one can prove a version for submanifolds contained in a horocylinder of $N \times L$, where $\operatorname{Sec}_{L} \geq-B$, under suitably modified assumptions on the dimensions.

Theorem 1.3. Let $\left(N, g_{N}\right)$ be an n-dimensional Cartan-Hadamard manifold with sectional curvature satisfying $-B \leq \operatorname{Sec}_{N} \leq-A<0$ where $A, B$ are positive constants. Let $f: \Sigma \hookrightarrow N$ be an m-dimensional submanifold properly immersed with $f(M)$ inside a horoball of $N$ and $n \leq 2 m-1$ then the sectional curvature of $\Sigma$ satisfies the estimate

$$
\sup _{\Sigma} \operatorname{Sec}_{\Sigma} \geq A-B
$$

As the geometric setting suggests, the main tool to obtain the results is the analysis of the Busemann function in Cartan-Hadamard manifolds which will be described in the next Section.

\section{BUSEMANN FUnCTIONS IN CARTAN-HADAMARD SPACES}

Throughout this section we let $\left(N, g_{N}\right)$ be a Cartan-Hadamard manifold, i.e., a simply connected, complete Riemannian manifold of non-positive sectional curvature. Further assumptions on $N$ will be introduced when required. First, we are going to collect some of the basic differentiable properties of the Busemann function of $N$ with respect to a fixed geodesic ray. Since we are not aware of any specific reference we decided to provide fairly detailed proofs.

Let $\sigma:[0,+\infty) \rightarrow N$ be a geodesic ray issuing from $\sigma(0)=o$. Recall that, by its very definition, the Busemann function of $N$ with respect to $\sigma$ is the function $b_{\sigma}: N \rightarrow \mathbb{R}$ defined by

$$
b_{\sigma}(x)=\lim _{t \rightarrow+\infty} b_{\sigma(t)}(x)
$$

where, for any fixed $t \geq 0$,

$$
b_{\sigma(t)}(x)=r_{\sigma(t)}(x)-r_{\sigma(t)}(o)=r_{\sigma(t)}(x)-t .
$$

Here and below, $r_{p}(x)=d(p, x)$ denotes the distance function from a point $p$. In some sense, the Busemann function measures the distance of the points of $N$ from the point $\sigma(+\infty)$ in the ideal boundary $\partial N$. Since $t \longmapsto$ $b_{\sigma(t)}(x)$ is monotone decreasing and bounded by $\left|b_{\sigma(t)}(x)\right| \leq r_{o}(x)$, the limit $b_{\sigma}(x)$ exists and is finite. Moreover, the convergence is uniform on compacts by Dini's theorem. Clearly, by the triangle inequality, each $b_{\sigma(t)}$ is 1-Lipschitz (in fact, $\left|\nabla b_{\sigma(t))}\right|=1$ by the Gauss Lemma) and, therefore, so is also $b_{\sigma}$. In particular, $b_{\sigma}$ is differentiable a.e. Actually, in the special case of Cartan-Hadamard manifolds it was proved by P. Eberlein, [5], that $b_{\sigma}$ is 
a function of class $C^{2}$. To begin with we observe that the gradient $\nabla b_{\sigma}$ of the Busemann function can be obtained via a limit procedure from $\nabla b_{\sigma(t)}$ as $t \rightarrow+\infty$.

Lemma 2.1 (limit of gradients). Assume that the sectional curvature of $N$ is bounded, namely, there exists $B>0$ such that $-B \leq \operatorname{Sec}_{N} \leq 0$. Then, for every $x \in N$,

$$
\lim _{t \rightarrow+\infty} \nabla b_{\sigma(t)}(x)=\nabla b_{\sigma}(x)
$$

and the convergence is locally uniform.

Proof. By the Hessian comparison theorem, [10], we know that, having fixed a compact ball $K \subset N$, there exist $T=T(K)>0$ and $C=C(K, B)>0$ such that

$$
\left|\operatorname{Hess} b_{\sigma(t)}\right| \leq C
$$

for every $x \in K$ and for every $t \geq T$. It follows that for any sequence $\left\{t_{k}\right\} \rightarrow$ $+\infty$ the corresponding sequence of gradients $\left\{\nabla b_{\sigma\left(t_{k}\right)}\right\}$ is eventually equicontinuous on $K$. Since it is equi-bounded as observed above, we deduce that there exists a subsequence $\left\{\nabla b_{\sigma\left(t_{k_{j}}\right)}\right\}$ that converges uniformly on $K$ to a continuous vector field $\xi$ on $K$. On the other hand, the sequence $\left\{\nabla b_{\sigma\left(t_{k}\right)}\right\}$ converges weakly to $\nabla b_{\sigma}$ on compact sets. Indeed, if $V$ is a smooth vector field supported in a ball $B_{R}$, then, by dominated convergence,

$$
\int_{B_{R}}\left\langle\nabla b_{\sigma\left(t_{k}\right)}, V\right\rangle=-\int_{B_{R}} b_{\sigma\left(t_{k}\right)} \operatorname{div} V \rightarrow-\int_{B_{R}} b_{\sigma} \operatorname{div} V=\int_{B_{R}}\left\langle\nabla b_{\sigma}, V\right\rangle,
$$

as claimed. It follows that

$$
\xi=\nabla b_{\sigma}
$$

a.e. on $K$ and, in fact, everywhere on $K$ by continuity. Since the selected sequence $\left\{t_{k}\right\}$ was arbitrary, the required conclusion follows.

In the above proof the conclusion is obtained using the weak definition of the gradient, which behaves well under limits, together with the fact the weak gradient agrees with the classical gradient for sufficiently regular functions. A similar trick will be used in the next result where we deduce a comparison principle for the Hessian of the Busemann function. We put the following:

Definition 2.2. A function $h: N \rightarrow \mathbb{R}$ is said to satisfy the differential inequality

$$
\text { Hess } h \leq \mathscr{T}
$$

in the sense of distributions, where $\mathscr{T}$ is a symmetric 2-tensor, if the integral inequality

$$
\int_{N} h\left\{\operatorname{div}(V \operatorname{div} V)+\operatorname{div} D_{V} V\right\} \leq \int_{N} \mathscr{T}(V, V)
$$


holds for every smooth compactly supported vector field $V$.

Clearly, in case $h$ is of class $C^{2}$, a double integration by parts shows that the distributional inequality is equivalent to

$$
\int_{N} \operatorname{Hess} h(V, V) \leq \int_{N} \mathscr{T}(V, V) .
$$

The validity of this latter for every compactly supported vector field $V$, in turn, is equivalent to the pointwise inequality. Indeed, suppose

$$
\operatorname{Hess}_{x} h(v, v)>\mathscr{T}_{x}(v, v),
$$

for some $x \in M$ and some $v \in T_{x} M \backslash\{0\}$. Extend $v$ to any smooth vector field $V^{\prime}$ on $M$. By continuity, there exists a neighborhood $\mathscr{U}$ of $x$ such that,

$$
\operatorname{Hess} h\left(V^{\prime}, V^{\prime}\right)>\mathscr{T}\left(V^{\prime}, V^{\prime}\right) \text {, on } \mathscr{U} \text {. }
$$

To conclude, choose a smooth cut-off function $\xi: M \rightarrow \mathbb{R}$ such that supp $\xi \subset$ $\mathscr{U}$ and $\xi=1$ at $x$, and observe that $V=\xi V^{\prime}$ violates the distributional inequality.

Lemma 2.3 (Hessian comparison). Assume that the sectional curvatures of $N$ satisfy

$$
-B \leq \operatorname{Sec}_{N} \leq-A
$$

for some constants $B \geq A>0$. Then

$$
\sqrt{A}\left(g_{N}-d b_{\sigma} \otimes d b_{\sigma}\right) \leq \operatorname{Hess} b_{\sigma} \leq \sqrt{B}\left(g_{N}-d b_{\sigma} \otimes d b_{\sigma}\right),
$$

in the sense of quadratic forms.

Proof. Let us show how to prove the upper bound of Hess $b_{\sigma}$. Obviously, the lower bound can be obtained using exactly the same arguments. By the Hessian comparison theorem, having fixed a ball $B_{R}$ of $N$, we find $T>0$ such that, for every $t \geq T$,

$$
\text { Hess } b_{\sigma(t)} \leq \sqrt{B} \operatorname{coth}\left(r_{\sigma(t)} \sqrt{B}\right)\left\{\left(g_{N}-d b_{\sigma(t)} \otimes d b_{\sigma(t)}\right)\right\} \text {, on } B_{R} \text {. }
$$

In particular, this inequality holds in the sense of distribution, namely, for every vector field $V$ compactly supported in $B_{R}$, it holds

$$
\int_{N} b_{\sigma(t)}\left\{\operatorname{div}(V \operatorname{div} V)+\operatorname{div} D_{V} V\right\} \leq \int_{N}\left\{|V|^{2}-\left\langle\nabla b_{\sigma(t)}, V\right\rangle^{2}\right\} .
$$

Evaluating this latter along a sequence $\left\{t_{k}\right\} \rightarrow+\infty$, using Lemma 2.1, and applying the dominated convergence theorem we deduce that the integral inequality

$$
\int_{N} b_{\sigma}\left\{\operatorname{div}(V \operatorname{div} V)+\operatorname{div} D_{V} V\right\} \leq \int_{N}\left\{|V|^{2}-\left\langle\nabla b_{\sigma}, V\right\rangle^{2}\right\}
$$


holds for every smooth vector field compactly supported in $B_{R}$. To conclude we now recall that this is equivalent to the pointwise inequality

$$
\text { Hess } b_{\sigma} \leq \sqrt{B}\left(g_{N}-d b_{\sigma} \otimes d b_{\sigma}\right)
$$

on $B_{R}$.

We remark that a version of the above lemma was also observed without proof in [4].

Corollary 2.4. Keeping the notation and the assumptions of Lemma 2.3 let $u: N \rightarrow \mathbb{R}$ be the smooth function defined by

$$
u(x)=e^{\sqrt{A} b_{\sigma}(x)}
$$

Then

$$
A u \cdot g_{N} \leq \operatorname{Hess} u \leq \sqrt{A B} u \cdot g_{N}
$$

\section{PROOF OF THE MAIN THEOREMS}

We are now ready to give the proof of our results.

Proof of Theorem 1.1 Let

$$
w=u \circ f_{N}: \Sigma \rightarrow \mathbb{R}_{>0}
$$

where $u: N \rightarrow \mathbb{R}$ is the smooth function defined in Corollary 2.4. By the composition law for the Laplacians we have

$$
\Delta w=\operatorname{tr}_{\Sigma}\left\{\operatorname{Hess} u\left(d f_{N} \otimes d f_{N}\right)\right\}+d u\left(\operatorname{tr}_{\Sigma} D d f_{N}\right) .
$$

On the other hand, by Corollary 2.4.

$$
\text { Hess } u \geq A u \cdot g_{N}
$$

so that

$$
\begin{aligned}
\Delta w & \geq w\left\{A \operatorname{tr}_{\Sigma} g_{N}\left(d f_{N} \otimes d f_{N}\right)-m \sqrt{A}|\mathbf{H}|\right\} \\
& =w\left\{A \operatorname{tr}_{\Sigma} f_{N}^{*}\left(g_{N}\right)-m \sqrt{A}|\mathbf{H}|\right\} .
\end{aligned}
$$

Since $f^{*} g_{N \times L}=g_{\Sigma}$ then

$$
\begin{aligned}
\operatorname{tr}_{\Sigma} f_{N}^{*}\left(g_{N}\right) & =m-\operatorname{tr}_{\Sigma} f_{L}^{*}\left(g_{L}\right) \\
& \geq m-\ell,
\end{aligned}
$$

and from the above we conclude that

$$
\Delta w(x) \geq m w(x)\left\{A \frac{m-\ell}{m}-\sqrt{A} \sup _{\Sigma}|\mathbf{H}(x)|\right\} .
$$

Now we apply the weak maximum principle for the Laplacian, [11], to get

$$
0 \geq m \sup _{\Sigma} w\left\{A \frac{m-\ell}{m}-\sqrt{A} \sup _{\Sigma}|\mathbf{H}(x)|\right\},
$$


as required.

Remark. By applying the strong maximum principle to (2) we can also obtain directly the following touching principle.

Let $f: \Sigma \hookrightarrow N \times L$ be a complete, immersed submanifold, where $N$ is a Cartan-Hadamard manifold of pinched negative curvature and $L$ is any complete Riemannian manifold. Let us assume that (a) the mean curvature $\mathbf{H}$ of the immersion satisfies $|\mathbf{H}| \leq \sqrt{A}(m-\ell) / m$ and that (b) $f(\Sigma)$ is contained inside the horocylinder $\mathscr{B}_{\sigma, R}^{n, \ell}$ and $f(\Sigma) \cap \partial \mathscr{B}_{\sigma, R}^{n, \ell} \neq 0$. Then $f(\Sigma)=\partial \mathscr{B}_{\sigma, R}^{n, \ell}$.

Remark. In a different direction, if $f=f_{N}: \Sigma \rightarrow \mathscr{B}_{\sigma, R}^{n, 0}=\left\{b_{\sigma} \leq R\right\} \subset N$ is a proper immersion into a horoball of $N$ and

$$
\sup _{\Sigma}|\mathbf{H}(x)|<\sqrt{A}
$$

then $w$ is a bounded exhaustion function that violates the weak maximum principle at infinity. By Theorem 32 in [3] it follows that the essential spectrum of $\Sigma$ is empty.

The estimates for the Hessian of the Busemann function allows us to obtain also the Jorge-Koutroufiotis type result stated in Theorem 1.3 . This results give a further indication of the phenomenon according to which submanifolds of non-compact horoballs behave in many respects like a submanifolds of compact balls.

Proof (of Theorem 1.3). The proof is similar to the arguments in [2]. For every $k$ consider the function $h_{k}: \Sigma \rightarrow \mathbb{R}$

$$
h_{k}=w-\frac{1}{k}\left[\log \left(\rho_{N} \circ f+1\right)+1\right]
$$

where, as above $w=e^{\sqrt{A} b_{\sigma}} \circ f$ and $\rho_{N}$ denotes the Riemannian distance in $N$ from an origin $o$ in the complement of $\mathscr{B}_{\sigma, R}^{n, 0}$. Since $f(\Sigma)$ is contained in a horoball, the first summand is bounded above, and since the $f$ is proper in $N$, the second summand tends to infinity at infinity. It follows that for every $k, h_{k}$ attains an absolute maximum at a point $x_{k}$ where

$$
\operatorname{Hess} h_{k}=\operatorname{Hess} w-\frac{1}{k} \operatorname{Hess}\left[\log \left(\rho_{N} \circ f+1\right)+1\right] \leq 0
$$

in the sense of quadratic forms. Now, according to our previous computations, for all vectors $X_{k} \in T_{x_{k}} \Sigma$,

$$
\operatorname{Hess} w\left(X_{k}, X_{k}\right) \geq \sqrt{A} w\left(x_{k}\right)\left(\sqrt{A}\left|X_{k}\right|^{2}-\left|\operatorname{II}\left(X_{k}, X_{k}\right)\right|\right),
$$


where $I I$ is the second fundamental form of the immersion. On the other hand, by the Hessian comparison theorem,

$$
\operatorname{Hess} \rho_{N} \leq \sqrt{B} \operatorname{coth}\left(\sqrt{B} \rho_{N}\right)\left(g_{N}-d \rho_{N} \otimes d \rho_{N}\right),
$$

and after some computation we obtain

$$
\begin{aligned}
\operatorname{Hess}( & {\left.\left[\log \left(\rho_{N} \circ f+1\right)+1\right]\right)\left(X_{k}, X_{k}\right) } \\
& \leq \frac{1}{\rho_{N}\left(f\left(x_{k}\right)\right)+1}\left\{\sqrt{B} \operatorname{coth}\left(\sqrt{B} \rho_{N}\left(f\left(x_{k}\right)\right)\right)\left|X_{k}\right|^{2}+\left|\operatorname{II}\left(X_{k}, X_{k}\right)\right|\right\}
\end{aligned}
$$

Combining the two inequalities and rearranging we conclude that

$$
\begin{aligned}
\left|\mathrm{II}\left(X_{k}, X_{k}\right)\right|\left(\sqrt{A} w\left(x_{k}\right)+\right. & \left.\frac{1}{k\left[\rho_{N}\left(f\left(x_{k}\right)\right)+1\right]}\right) \\
& \geq\left(A w\left(x_{k}\right)-\frac{\sqrt{B} \operatorname{coth}\left(\sqrt{B} \rho_{N}\left(f\left(x_{k}\right)\right)\right)}{k\left[\rho_{N}\left(f\left(x_{k}\right)\right)+1\right]}\right)\left|X_{k}\right|^{2}
\end{aligned}
$$

Now notice that $w\left(x_{k}\right)$ is positive and bounded away from 0 . Indeed, if $\bar{x}$ is a point such that $\rho_{N}(f(\bar{x}))=\min _{\Sigma} \rho_{N}(f(x))$, then for every $k$ we have

$$
h_{k}\left(x_{k}\right) \geq h_{k}(\bar{x})
$$

and therefore

$$
w\left(x_{k}\right) \geq w(\bar{x})+\frac{1}{k}\left\{\log \left[\rho_{N}\left(f\left(x_{k}\right)\right)+1\right]-\log \left[\rho_{N}(f(\bar{x}))+1\right]\right\} \geq w(\bar{x}) .
$$

Since $\rho_{N}\left(f\left(x_{k}\right)\right)$ is also bounded away from zero, it follows that for every sufficiently large $k$, and every non zero $X_{k} \in T_{x_{k}} \Sigma$,

$$
\begin{aligned}
\left|\mathrm{II}\left(X_{k}, X_{k}\right)\right| & \geq \frac{A w\left(x_{k}\right)-\frac{\sqrt{B} \operatorname{coth}\left(\sqrt{B} \rho_{N}\left(f\left(x_{k}\right)\right)\right)}{k\left[\rho_{N}\left(f\left(x_{k}\right)\right)+1\right]}}{\sqrt{A} w\left(x_{k}\right)+\frac{1}{k\left[\rho_{N}\left(f\left(x_{k}\right)\right)+1\right]}}\left|X_{k}\right|^{2} \\
& =\left[\sqrt{A}+o\left(k^{-1}\right)\right]\left|X_{k}\right|^{2}
\end{aligned}
$$

In particular, $\operatorname{II}\left(X_{k}, X_{k}\right)>0$ for every sufficiently large $k$ and every $X_{k} \in$ $T_{x_{k}} \Sigma \backslash\{0\}$ and we may apply Otsuki's lemma (see, e.g., [9], p. 28) to find unit tangent vectors $X_{k}$ and $Y_{k}$ such that $\operatorname{II}\left(X_{k}, X_{k}\right)=\operatorname{II}\left(Y_{k}, Y_{k}\right)$ and $\mathrm{II}\left(X_{k}, Y_{k}\right)=0$. The required conclusion now follows from Gauss equations as in the original proof:

$$
\begin{aligned}
\operatorname{Sec}_{\Sigma}\left(X_{k}, Y_{k}\right) & =\operatorname{Sec}_{N}\left(d f X_{k}, d f Y_{k}\right)+g_{N}\left(\operatorname{II}\left(X_{k}, X_{k}\right), \operatorname{II}\left(Y_{k}, Y_{k}\right)\right)-\left|\operatorname{II}\left(X_{k}, Y_{k}\right)\right|^{2} \\
& \geq-B+A+o\left(k^{-1}\right) .
\end{aligned}
$$


Again as in the classical proof, we note that the conclusion of the theorem follows directly from (3) if we assume that the weak maximum principle for the Hessian holds, for then there exists a sequence $x_{k}$ such that $w\left(x_{k}\right) \rightarrow$ $\sup _{\Sigma} w$ and

$$
\operatorname{Hess} w\left(x_{k}\right) \leq 1 / k g_{\Sigma}
$$

which together with (3) allows to conclude as in the last part of the above proof. In particular, the conclusion holds if $\operatorname{Scal}_{\Sigma} \geq-G\left(\rho_{\Sigma}\right)$ where the function $G$ is positive, non-decreasing and $G^{-1 / 2}$ is integrable at infinity. Indeed, assuming that $\operatorname{Sec}_{\Sigma}$ is bounded above, for otherwise the conclusion holds trivially, then $\operatorname{Sec}_{\Sigma}$ is bounded below by a multiple of $-G$ and the Omori-Yau maximum principle for the Hessian holds on $\Sigma([12])$.

\section{REFERENCES}

[1] L. J. Alias , G.P. Bessa, M. Dajczer, The mean curvature of cylindrically bounded submanifolds. Math. Ann. 345 (2009), 367-376.

[2] G. P. Bessa, B P. Lima, L. F. Pessoa, Curvature estimates for properly immersed $\phi_{h}$-bounded submanifolds. Ann. Mat. Pura Appl., to appear. arXiv: 1211.5910, DOI 10.1007/s10231-013-0367-1

[3] G. P. Bessa, S. Pigola, A. G. Setti, Spectral and stochastic properties of the $f$ Laplacian, solutions of PDEs at infinity and geometric applications. Rev. Mat. Iberoam. 29 (2013), 579-610.

[4] M. P. Cavalcante, F. Manfio, Lower bound estimates for the first eigenvalue of the Laplacian on complete submanifolds. arXiv:1306.1559.

[5] E. Heintze, H.C. Im Hof, Geometry of horospheres. Jour. Diff. Geom. 12 (1977), 481491.

[6] L. Jorge, D. Koutroufiotis, An estimate for the curvature of bounded submanifolds. Amer. J. Math. 103 (1981), 711-725.

[7] L. Jorge, and F. Xavier, An inequality between the exterior diameter and the mean curvature of bounded immersions. Math. Z. 178, 77-82, (1981)

[8] L. Karp, Differential inequalities on complete riemannian manifolds and applications. Math. Ann. 272 (1985), 449-459.

[9] S. Kobayashi, K. Nomizu, Foundations of Differential Geometry, Vol II. Wiley Classics Library, Wiley-Interscience. New York 1996.

[10] P. Petersen, Riemannian geometry. Second edition. Graduate Texts in Mathematics, 171. Springer, New York, 2006.

[11] S. Pigola, M. Rigoli, A. G. Setti, A remark on the maximum principle and stochastic completeness. Proc. Amer. Math. Soc. 131 (2003), 1283-1288.

[12] S. Pigola, M. Rigoli, A. G. Setti, Maximum Principles on Riemannian Manifolds and Applications. Memoirs of the American Mathematical Society, vol. 174, no. 822 (2005). American Mathematical Society - AMS, Providence, Rhode Island. 
10G. PACELLI BESSA, JORGE H. DE LIRA, STEFANO PIGOLA, AND ALBERTO G. SETTI

Departamento de Matemática, Universidade Federal do Ceará-UFC, 60455-760 ForTALEZA, CE, BRAZIL.

Departamento de Matemática, Universidade Federal do Ceará-UfC, 60455-760 ForTALEZA, CE, BRAZIL.

Sezione di Matematica - DiSAT, Universitá dell'Insubria - Como, Via VAllegGio 11, I-22100 Como, ITALY

Sezione di MAtematicA - DisAT, Universitá Dell'insubria - Como, Via VALLEGGIO 11, I-22100 COMO, ITALY 